\title{
In-Situ 2p3d Resonant Inelastic X-ray Scattering Tracking Cobalt Nanoparticle Reduction
}

\author{
Boyang Liu, ${ }^{\dagger}$ Matti M. van Schooneveld, ${ }^{\dagger}$ Yi-Tao Cui, ${ }^{\ddagger}$ Jo Jun Miyawaki, ${ }^{\ddagger}$ Yoshihisa Harada, ${ }^{\ddagger}$ \\ Thomas O. Eschemann, ${ }^{\dagger}$ Krijn P. de Jong, ${ }^{\dagger}$ Mario U. Delgado-Jaime, ${ }^{, \dagger}$ and Frank M. F. de Groot ${ }^{* \dagger}{ }^{\dagger}$ \\ ${ }^{\dagger}$ Inorganic Chemistry \& Catalysis, Debye Institute for Nanomaterials Science, Utrecht University, Universiteitsweg 99, Utrecht, 3584 \\ CG, The Netherlands \\ ${ }^{\ddagger}$ Institute for Solid State Physics, University of Tokyo, Kashiwa, Chiba 277-8581, Japan
}

Supporting Information

ABSTRACT: In-situ carbon-thermal reduction of cobalt oxide nanoparticles supported on carbon nanotubes was studied by cobalt 2 p3d resonant inelastic X-ray scattering (RIXS). The in-situ 2p X-ray absorption spectroscopy (XAS) and RIXS measurements were performed at 500,600 , and $700{ }^{\circ} \mathrm{C}$, where four consistent excitation energies were used for RIXS acquisitions. After $700{ }^{\circ} \mathrm{C}$ reduction, the XAS spectrum shows a cobalt metal-like shape, while the RIXS spectra reveal the minority cobalt monoxide phase. The holistic fit on both XAS and RIXS data reveals the respective contributions from metal and monoxide. We show that the relative precision to determine the monoxide content changes from $\sim 5.6 \%$ in XAS results to better than $0.8 \%$ in the RIXS analysis, suggesting that RIXS is a useful tool to track the oxidation state of nanoparticles under in situ conditions. We determined a relative radiative ratio $(P)$ factor of approximately 5 , where this factor gives the ratio between the relative strengths of the radiative decay channels compared to the nonradiative channels in $\mathrm{CoO}$ and $\mathrm{Co}$ metal.

\section{INTRODUCTION}

In chemical reactions where transition metal nanoparticles play pivotal roles, it is an important goal to track the phase evolution of nanoparticles during activation and usage. In catalysis, for example, tracking chemical phase changes can be essential to unveil deactivation processes of catalysts, while quantifying the active phase can be used to understand catalytic performances. ${ }^{1}$ A nuisance of probing minority chemical species is that they are often obscured by signal of majority phases. A minority phase in the sea of major chemical phases can, however, be a key phase, such as the catalytic active phase, that requires the effective detection of it. X-ray absorption spectroscopy (XAS) is an element-specific technique that is sensitive to the oxidation state and different chemical environments. In-situ XAS further allows the probing of phase information under relevant reaction conditions. ${ }^{2-4}$ Due to strong multiplet effects and a small corehole lifetime broadening, ${ }^{5}$ p XAS spectra ( $\mathrm{L}_{2,3}$ edges) of transition metals exhibit rich characteristic features and high chemical sensitivities. Because the XAS edge jump is an atomic property, the XAS intensity scales linearly with the number of atoms, which has made XAS a widely applied technique to discriminate phase heterogeneities and to obtain phase concentrations by fitting procedures. ${ }^{6-9}$ However, for minority phase detection, a limitation present in 2 p XAS is that signals from majority phases overwhelm details of the minority phase. $^{10}$ Although fitting procedures were used to determine the minority phase information including concentrations, we note that the overlap of spectroscopic signatures from different phases can lead to multiple interpretations. A minority phase, under such cases, can even be undetected when its signatures are hidden under those from majority phases. In this regard, high-energy resolution 2p3d resonant inelastic X-ray scattering (RIXS), which can selectively enhance spectral features from a particular phase of interest, is a complementary spectroscopic tool to $2 \mathrm{p}$ XAS. Because the RIXS signal can selectively be enhanced by the corresponding XAS intensity, it is a nonlinear technique with respect to the number of atoms that adds to the possible detection of minority phases.

We use $\mathrm{Co}_{3} \mathrm{O}_{4}$ nanoparticles supported on CNTs as model Fischer-Tropsch catalyst precursors, where we study the reduction process of $\mathrm{Co}_{3} \mathrm{O}_{4}$ nanoparticles. We show that $2 \mathrm{p} 3 \mathrm{~d}$ RIXS can be more useful than $2 \mathrm{p}$ XAS in detecting the minority species under in-situ conditions. In an ex-situ study the higher accuracy of $2 \mathrm{p} 3 \mathrm{~d}$ RIXS over $2 \mathrm{p}$ XAS in detecting minority species was previously demonstrated. ${ }^{10}$ Here we perform detailed fits to a large data set, acquired in situ, to reveal more insights. The fitted results are used to understand the sensitivity of 2p3d RIXS on the minority oxide phase detection. Moreover, the established fitting approach allows the quantitative description of a mixed-phase system with high precision, revealing application potential in the study of active catalysts and other active materials.

Received: May 6, 2017

Revised: July 10, 2017

Published: July 14, 2017 


\section{EXPERIMENTS}

The $\mathrm{Co}_{3} \mathrm{O}_{4} / \mathrm{CNT}$ catalyst was prepared according to published procedures $^{11}$ with a cobalt loading of $8 \mathrm{wt} \%$. The dark field transmission electron microscopy (TEM) of the fresh catalyst (Figure 1A) shows an average particle size around $5 \mathrm{~nm}$. Some
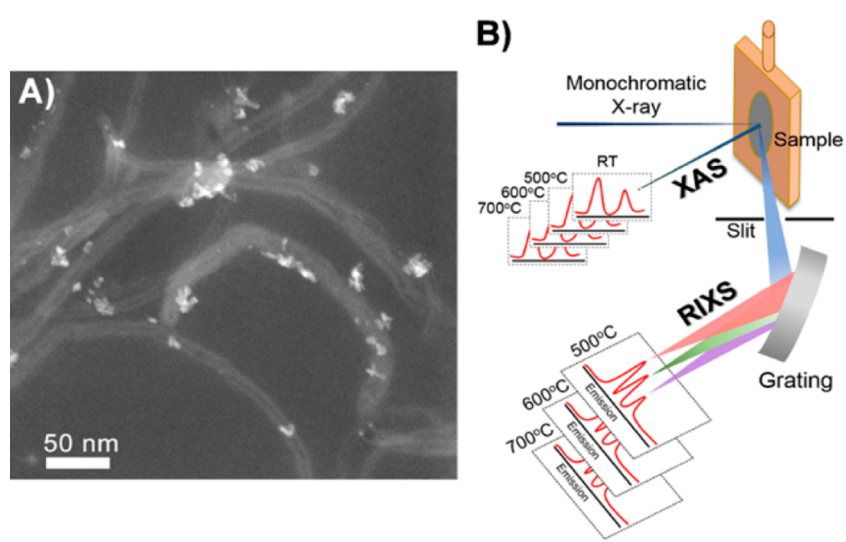

Figure 1. (A) Dark-field TEM image of the fresh catalyst. (B) Schematic representation of XAS and RIXS measurements.

nanoparticle agglomerates are visible, and in the agglomerates it is in principle possible that there will be two $\sim 5 \mathrm{~nm}$ nanoparticles behind each other in the line of the X-ray beam. This would generate effectively a $10 \mathrm{~nm}$ beam path through the particles. Given the X-ray penetration depth of $\sim 50$ $\mathrm{nm}$, this will not yield any saturation effect for X-rays. The electron yield has an escape depth of $\sim 5 \mathrm{~nm}$, and minor saturation effects could occur; however, due to the majority of 5 $\mathrm{nm}$ nanoparticles this will not be detectable. Both X-ray powder diffraction (XRD) and cobalt 2p XAS, shown in the Supporting Information, indicate the $\mathrm{Co}_{3} \mathrm{O}_{4}$ phase of cobalt oxide nanoparticles before reduction.

In Fischer-Tropsch synthesis, cobalt metal is reported to be the active phase during hydrogenation of CO. ${ }^{2,13}$ Several studies have applied in-situ TEM or XRD to examine the reduction process of carbon-supported transition metal nanoparticle catalysts. ${ }^{14-16}$ However, TEM has a limitation of probing phase information only to selected nanoparticles; XRD reveals crystalline phases only in nonquantitative ways. On the contratry, X-ray spectroscopies offer bulk phase information on nanoparticles with feasibilities to quantify phase concentrations. Here we compare differences of in-situ $2 p$ XAS and 2p3d RIXS for revealing the relative phase composition of cobalt-based nanoparticles during reduction.

In-situ measurements were performed at BL07LSU ${ }^{17}$ in SPring- 8 . The catalyst was pressed into a thin pellet and loaded on the sample holder, which was then heated in a vacuum chamber attached next to the main chamber for the sample reduction. The temperature on the sample surface was monitored by a well-calibrated pyrometer. After heating in vacuum at 500,600 , and $700{ }^{\circ} \mathrm{C}$, respectively, the sample was cooled and transferred into the main chamber for spectra acquisitions. Figure 1B sketches the XAS and RIXS measurements. XAS spectra were collected with the total electron yield mode by measuring the sample current. RIXS spectra were acquired under a $90^{\circ}$ scattering angle in the linear horizontal scattering geometry. Detailed experimental procedures and spectra acquisitions are specified in the Supporting Information.

\section{RESULTS AND DISCUSSION}

Temperature-dependent cobalt $2 \mathrm{p}_{3 / 2}$ XAS spectra of the catalyst together with literature reference $\mathrm{CoO}^{18}$ and cobalt metal $^{19}$ spectra are shown in Figure 2A. From the analysis, it is clear that $\mathrm{CoO}$ and cobalt metal phases coexist in cobalt nanoparticles after reduction at 500 and $600{ }^{\circ} \mathrm{C}$. No sign of the initial $\mathrm{Co}_{3} \mathrm{O}_{4}$ is observed after these treatments. The $\mathrm{CoO}$ phase concentration decreased with increasing reduction temperature, as shown by the drop of relative intensities of features at the pre-edge $(777.3 \mathrm{eV})$ and the shoulder $(779.8$ $\mathrm{eV})$. The spectrum acquired after the reduction at $700{ }^{\circ} \mathrm{C}$ shows no visible fine structures of $\mathrm{CoO}$, from which it could then be erroneously concluded that the fully reduced cobalt
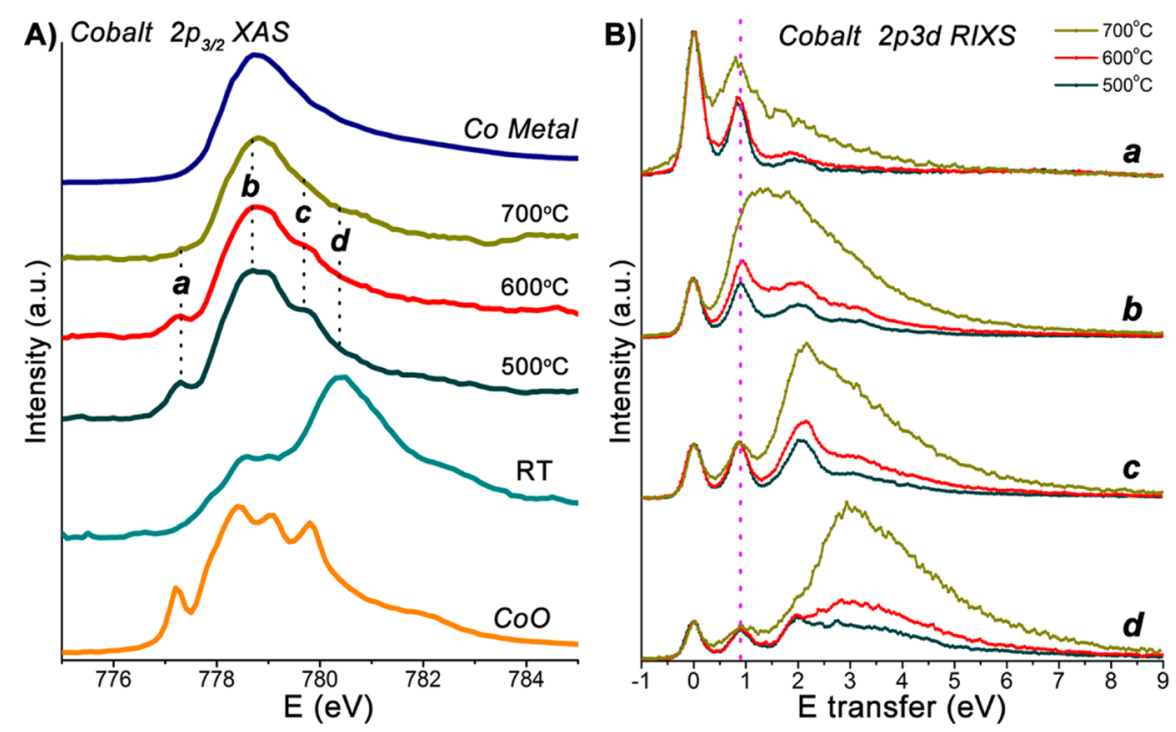

Figure 2. Experimental cobalt spectra of the catalyst after vacuum heating at room temperature, 500,600 , and $700{ }^{\circ} \mathrm{C}:(\mathrm{A}) 2 \mathrm{p}_{3 / 2} \mathrm{XAS}$ spectra. Four dotted lines indicate excitation energies $a-d$ for 2 p3d RIXS. (B) 2p3d RIXS spectra at $500{ }^{\circ} \mathrm{C}$ (dark green), $600{ }^{\circ} \mathrm{C}$ (red), and $700{ }^{\circ} \mathrm{C}$ (dark yellow). The purple dotted line indicates the first $\mathrm{d}-\mathrm{d}$ excitation from $\mathrm{CoO}$. In (A), the literature reference spectra of $\mathrm{Co}^{18}$ and $\mathrm{CoO}^{19}$ are plotted for comparison. 


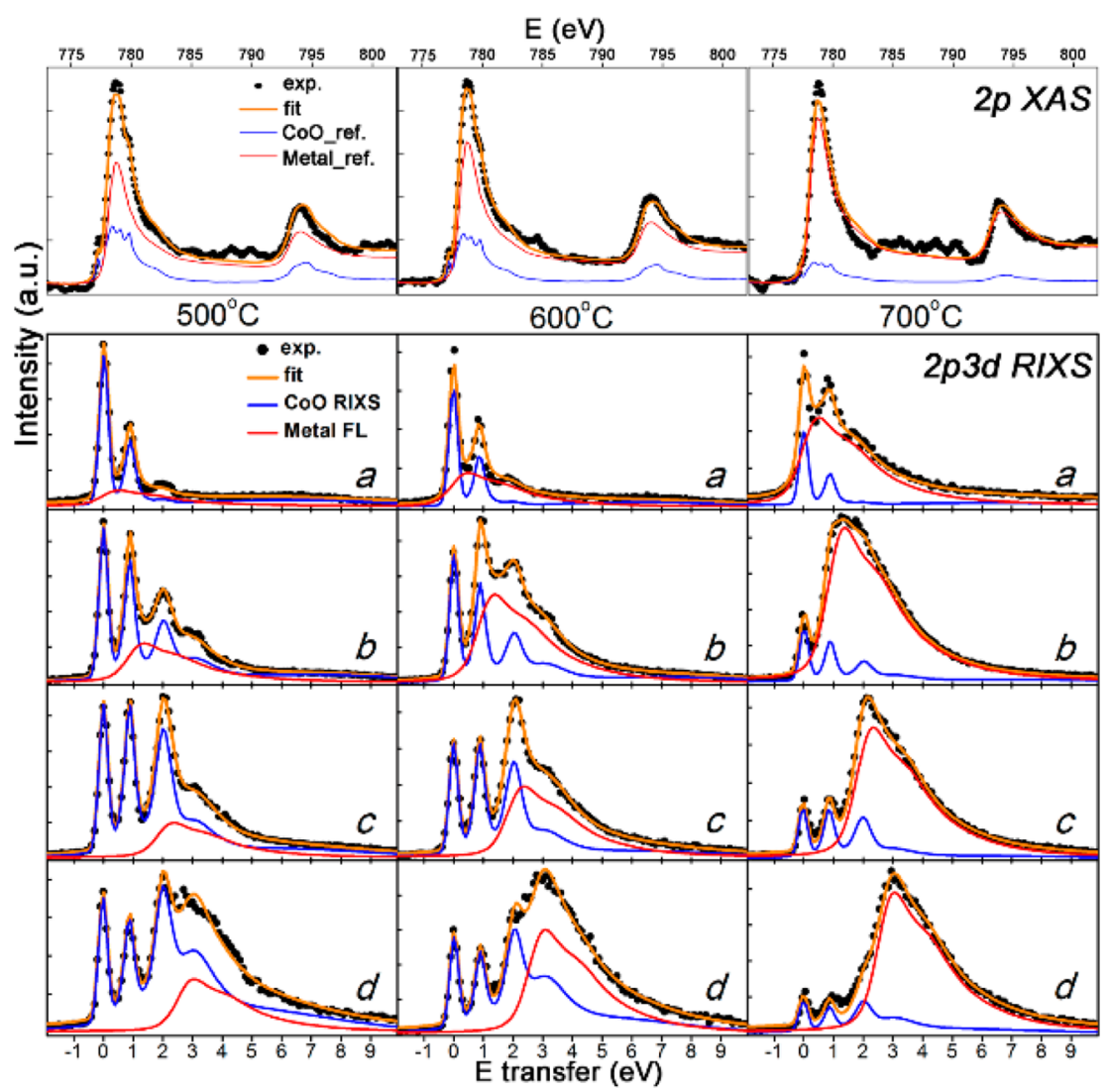

Figure 3. Fit (orange line) and experimental (black dot) cobalt 2p XAS (upper panel) and 2p3d RIXS (lower panel) spectra. Data sets of the same temperature are in the same column. CoO (blue line) and cobalt metal (red line) references used for fitting $2 \mathrm{p}$ XAS are literature reference spectra. ${ }^{18,19}$ In 2p3d RIXS, the CoO RIXS (blue line) and metal FL (red line) contributions are shown.

nanoparticles are present. Nevertheless, here in situ 2p3d RIXS was carried out to further examine the phases changes and map its complementariness to $2 \mathrm{p}$ XAS. For RIXS measurements, excitations were chosen at energies $a-d$ as indicated in Figure $2 \mathrm{~A}$.

2p3d RIXS spectra of metal oxides are composed of the zero energy transfer peak that corresponds to the elastic scattering and positive energy transfer features corresponding to phonon, magnon, $\mathrm{d}-\mathrm{d}$, and charge transfer excitations. ${ }^{20,21}$ Phonon and magnon excitations constitute low energy $(<0.1 \mathrm{eV})$ features, while $d-d$ excitations occur at the fixed energy transfer corresponding to energy differences with respect to the ground state irrespective of incident $\mathrm{X}$-ray energies. The charge transfer excitations in a divalent metal oxide, such as $\mathrm{CoO}$, have little intensity and are often shaded by $\mathrm{d}-\mathrm{d}$ features unless excitation energies at charge transfer satellites in 2 p XAS are chosen. ${ }^{18,22}$ For metals, the 2p3d RIXS measures a fixed X-ray emission by transition from delocalized states to the $2 p$ core hole that has energy transfer from the elastic line linearly dependent on the incident X-ray energies and is described as fluorescence (FL). ${ }^{23}$ Here the 2p3d RIXS signals originated from $\mathrm{CoO}$ or cobalt metal are referred to as "CoO RIXS" and "Co metal FL", respectively.

Figure $2 \mathrm{~B}$ shows the corresponding temperature-dependent 2p3d RIXS spectra. Data sets from the same excitation energy are normalized to the elastic peak. The increase of the pure metal concentration is most directly observed from spectra acquired at energy $d$, since the metal FL peak around $3 \mathrm{eV}$ separates further from $\mathrm{CoO}$ characteristic $\mathrm{d}-\mathrm{d}$ peaks around 0.9 and $2.1 \mathrm{eV}^{18,22}$ In the current reduction steps, no new phases other than the $\mathrm{CoO}$ and cobalt metal, such as cobalt carbides, were found, consistent with previously reported in situ $\mathrm{XRD}$ results. ${ }^{11,15}$ Interestingly, the $\mathrm{d}-\mathrm{d}$ peak of $\mathrm{CoO}$ around $0.9 \mathrm{eV}$ in data sets of $700{ }^{\circ} \mathrm{C}$ from excitation energies $a, c$, and $d$ unequivocally indicates the partially reduced nature of cobalt nanoparticles, contrary to the possible conclusion drawn from XAS. We note that the probing depth of TEY XAS at the cobalt $2 \mathrm{p}_{3 / 2}$ edge is $\sim 5 \mathrm{~nm},{ }^{24}$ while the RIXS can detect photons emitted from several hundreds of nanometers underneath the sample surface. ${ }^{25}$ Here, however, the applied heating method (see the Supporting Information) induces the homogeneous reduction of the sample, and the $\sim 5 \mathrm{~nm}$ (even smaller after reduction ${ }^{11}$ ) cobalt nanoparticles are fully probed by both techniques, but with different sensitivities. In 2p XAS, spectral features of the minority $\mathrm{CoO}$ phase are shaded by that of the majority metal phase. However, in 2p3d RIXS, d-d peaks of the CoO RIXS are dominantly enhanced at the excitation energy $a$ and separated from the metal FL when excitations move to higher energies, although at the metal resonance (energy $b$ ), oxide features are hidden under the metal FL. Using the difference in XAS intensity at different energy positions, we selectively use the corresponding RIXS features to enhance the $\mathrm{CoO}$ signal, making it more intense than the metal species.

To obtain phase concentrations and to further explore the sensitivity of $2 \mathrm{p} 3 \mathrm{~d}$ RIXS for revealing the minority $\mathrm{CoO}$ phase, fitting procedures ${ }^{26,27}$ were introduced for both XAS and RIXS spectra. Figure 3 shows the fitted spectra along with the corresponding experimental data and spectral components used for fitting. 
Temperature-dependent 2p XAS spectra (Figure 3, upper panel) were fitted holistically. The principle of the holistic fit was described in the literature. ${ }^{26}$ Here the $2 p$ XAS at 500, 600, and $700{ }^{\circ} \mathrm{C}$ were fitted by using literature $\mathrm{CoO}^{18}$ and cobalt metal ${ }^{19}$ spectra as references. In the fit model, a background function ${ }^{27}$ was included to fit both the raw experimental spectra and the two literature reference spectra. The reference spectra after background subtraction were normalized to the intensity of the post- $\mathrm{L}_{2}$ edge $(\sim 803 \mathrm{eV})$. For the two reference spectra used (between 773 and $803 \mathrm{eV}$ ), the $\mathrm{L}_{2,3}$ continuum edge jumps with the 2:1 ratio were included for the Co metal reference spectrum (see the literature reference ${ }^{19}$ ), while only the $\mathrm{L}_{3}$ continuum edge jump was set for the $\mathrm{CoO}$ reference spectrum. Here, the different edge jumps were set due to the difference of electron localization in oxides and in metal. Note that in the fits edge jump positions were allowed to float, which were also linked with the above-mentioned background functions. The scaling factors and the energy shifts between the reference spectra and experimental data were also set in the fit model, which together with other parameters were fitted simultaneously. Concentrations of the $\mathrm{CoO}$ phase obtained from XAS fitting are listed in Table 1 (second column). Note

Table 1. 2p XAS and 2p3d RIXS Derived Percentages of the CoO Phase in Co Nanoparticles during the Three Reduction Steps $^{a}$

$\begin{array}{cccc}\begin{array}{c}\mathrm{CoO} \\ \text { temperature } \\ \left({ }^{\circ} \mathrm{C}\right)\end{array} & \begin{array}{c}\text { concentration } \\ (\%) \text { from 2p XAS } \\ \text { fit }\end{array} & \begin{array}{c}\mathrm{CoO} \text { spectral } \\ \text { weight (\%) from } \\ \text { 2p3d RIXS fit }\end{array} & \begin{array}{c}\mathrm{CoO} \text { concentration } \\ (\%) \text { from 2p3d } \\ \text { RIXS fit }\end{array} \\ 500 & 18.4 \pm 6.1 & 56.9 \pm 2.5 & 20.9 \pm 1.7 \\ 600 & 14.2 \pm 6.0 & 33.1 \pm 1.3 & 9.0 \pm 0.5 \\ 700 & 5.5 \pm 4.6 & 10.7 \pm 0.8 & 2.3 \pm 0.2\end{array}$

${ }^{a_{T}}$ The concentration values from XAS are obtained directly from the corresponding spectral weights, as obtained from the fits, by normalizing the reference data for $\mathrm{CoO}$ and $\mathrm{Co}$ using $P=5$. Errors given as one standard deviation from all the good fits. Errors in column 4 are calculated from column 3 .

that although fitting results indicate the existence of a $\mathrm{CoO}$ phase after the reduction at $700{ }^{\circ} \mathrm{C}$ the $4.6 \%$ uncertainty with respect to a $5.5 \%$ average value implies the inadequacy of $2 p$ XAS alone for revealing precise information on this minority phase. In Figure S2 this is further elaborated upon, by showing a series of cobalt $2 p$ XAS spectra, consisting of small contributions of CoO XAS spectra added to pure Co metal XAS spectra. For $\mathrm{CoO}$ contents $<10 \%$ of the obtained high spectral similarities between them explain the large uncertainty in such fitting results.

The 12 2p3d RIXS data sets (Figure 3, lower panel) were fitted by using a separate model without including reference spectra. The 12 data sets were merged into one data set that allows the decomposition and fits to be performed holistically. Here, each experimental Co 2p3d RIXS spectrum was composed of "artificial" CoO RIXS and Co FL. All individual peaks that used to comprise the CoO RIXS and Co FL are the pseudo-Voigt peaks.

For the CoO RIXS, it is composed of an elastic peak, d-d peaks, and a separate peak that simulates the charge-transfer (CT) effects. The relative energy position of $d-d$ peaks was set to be constant in all the data sets, while energy positions of the CT peak were only fixed for data sets acquired at the same excitation energy. Similarly, all the elastic and $d-d$ peaks were forced to have the same shape (the Lorentzian:Gaussian ratio and the broadening of full width at half-maximum), while the CT peak is only set to be with the same shape for data sets belonging to the same excitation energy and which was allowed to float in the model. Furthermore, the relative intensities of all CoO RIXS features were kept constant for data sets collected at the same excitation energies, but this varies for data sets belonging to different excitation energies. The decomposefitted CoO RIXS was compared with the literature ${ }^{18}$ reference $\mathrm{CoO}$ (obtained with different energy resolutions), as shown in Figure S3. Apart from the excitation energy $a$, other excitation energies chosen in these two works were not consistent; hence, we could only compare RIXS slices obtained by close excitation energies, as noted in the figure legends. The RIXS slices were normalized to the first $\mathrm{d}-\mathrm{d}$ peak since the elastic peak intensity for data from different measurements is not directly comparable. The comparison of spectra at excitation energy $d$ is not shown in Figure S3 since no reference spectra acquired at close excitation energies are available.

For the Co FL, in this model, it was assumed to be with the same shape in all 12 data sets. Because in fluorescence the excitation is decoupled from the decay, the metal FL can be considered as the convolution of XAS with the X-ray emission spectra above the edge. When the excitation energy is chosen above the edge the XAS spectral shape is constant (or slowly varying in intensity) implying that the Co FL is also constant in shape. At the absorption edge the shape of the XAS spectrum slightly modifies the Co FL, where a peak maximum makes the shape sharper and a valley makes the shape slightly broader [see literature reference, ${ }^{28}$ cf Figure 1]. The fixed shape FL envelope as a whole consists of three peaks, whose relative energy positions, intensity ratios, and shapes are set to be constant. The energy shifts between FL envelopes, belonging to data sets acquired at excitation energies $b-d$, were set to the corresponding shifts of excitations. However, for the FL envelope that was acquired at the excitation energy $a$, its energy position was allowed to float since the fixed emission breaks down for this excitation energy. (Here, we need to better interpret our assumption.) The decompose-fitted Co FL was shown in Figure S4. Decompose-fitted results showed that the energy difference between the FL maxima from data sets acquired at excitation energies $a$ and $b$ was around $0.8 \mathrm{eV}$, whereas the difference between excitation energies $a$ and $b$ is $1.4 \mathrm{eV}$. The discrepancy is due to that the X-ray Raman was measured when choosing the excitation energy $a$, while at excitation energies $b-d$ the 2p3d RIXS of Co metal were pure FL with fixed emission energies.

Initially, the total CoO RIXS intensity (integrated area) was parametrized as a function of the total Co FL intensity, shown as eq 1

$$
r_{i j}=\frac{I_{\mathrm{FL}, i j}}{I_{\mathrm{RIXS}, i j}}
$$

where the parameter $r_{i j}$ is dependent on the temperature $i$ and the excitation energy $j$.

Since RIXS is a "photon-in photon-out" technique, the $r_{i j}$ was first normalized by the absorption cross-section ratio of $\mathrm{CoO}$ $\left(\sigma_{\mathrm{CoO}, \mathrm{j}}\right)$ to Co metal $\left(\sigma_{\mathrm{Co}, \mathrm{j}}\right)$, as shown in eq 2 . Here, this ratio was obtained from the normalized reference spectra shown in Figure 2A. The new parameter $R_{i}$ in this model is only dependent on temperature, which directly implies the ratio of spectra contribution by Co metal to CoO. 


$$
R_{i}=\frac{I_{\mathrm{FL}, i j}}{I_{\mathrm{RIXS}, i j}} \times \frac{\sigma_{\mathrm{CoO}, j}}{\sigma_{\mathrm{Co}, j}}
$$

Similar to the XAS fit, all parameters set in this decompositionfit model were fitted simultaneously by the Blueprint XAS. The results are chosen to be shown as the $\mathrm{CoO}$ contribution in Table 1.

In principle, RIXS is a more bulk sensitive technique than TEY XAS since the attenuation depth of the photon is much larger than electrons. Considering the $5 \mathrm{~nm}$ (even smaller after the reduction ${ }^{11}$ ) Co nanoparticles in the current studied materials, the TEY XAS is almost equivalent to the RIXS with respect to the detection depth. On the other hand, the XAS fitted results directly represent the percentage of the $\mathrm{CoO}$ and Co metal in the Co nanoparticles, whereas the RIXS fitted results only represent the spectra intensity contribution from either phase. To directly compare the XAS and RIXS fitted results, an extra factor that represents the signal yields a ratio of $\mathrm{CoO}$, and the Co metal phase for the respective RIXS and FL is further needed. With this factor, percentages of the $\mathrm{CoO}$ phase can be extracted from the RIXS fitted results, as shown in eq 3 . The signal yield is an intrinsic property of materials, and this ratio is therefore a constant $(P)$ between $\mathrm{Co}$ and $\mathrm{CoO}$. In eq 3, the $P$ represents the ratio of Co FL to $\mathrm{CoO}$ RIXS yield by the same unit of respective phases. Here, the constant $P$ in the equation is the relative radiation ratio as discussed above.

$$
\mathrm{CoO} \%=\frac{1}{R_{i} P+1} \times 100
$$

In the current work, we compared the $2 \mathrm{p}$ XAS and $2 \mathrm{p} 3 \mathrm{~d}$ RIXS fitted results at 500 and $600{ }^{\circ} \mathrm{C}$. We ignored the $2 \mathrm{p}$ XAS fitted results of $700{ }^{\circ} \mathrm{C}$ because the large uncertainty rendered a larger uncertainty without statistical meanings.

The quantification procedure was based on eqs 1 and 2, in which the intensity ratios between CoO RIXS and metal FL were corrected by the corresponding X-ray absorption cross section, to account for the photon-in and photon-out process of RIXS. In contrast to XAS, RIXS quantifications contain an extra relative radiative ratio related to the difference of the $\mathrm{Co} / \mathrm{CoO}$ spectral intensity yield as stated by eq 3 . This relative radiative ratio $(P)$ is given by the relative (integrated) decay strength of CoO RIXS compared with Co metal fluorescence, which determines the capability of 2p3d RIXS for revealing the minority $\mathrm{CoO}$ phase. By comparing the XAS and RIXS fitting results for the 500 and $600{ }^{\circ} \mathrm{C}$ data, we deduct that the $\mathrm{CoO}$ RIXS is approximately 5 times stronger than the Co metal fluorescence, implying a ratio $P=5$. The $700{ }^{\circ} \mathrm{C}$ data are not used as the uncertainty in the XAS is almost as large as the determined value. We note that the value $P=5$ cannot be determined with good accuracy, and we estimate its uncertainty as large as $20 \%$, based on the limited experiments performed for this goal. A dedicated experiment at various compositions needs to be performed in order to determine this number with more accuracy. Moreover, we note that the lifetime of a state is given by the integrated Auger decay because Auger constitutes more than $99 \%$ of the decay. The lifetime broadening of the $\mathrm{L}_{3}$ edge states is usually taken as constant based on calculations of all Auger decay channels for $\mathrm{NiO}$ that yield a variation smaller than $20 \%$ over the $\mathrm{L}_{3}$ edge. ${ }^{29}$ The lifetime broadening of cobalt metal could potentially be different from $\mathrm{CoO}$, where we note that experimental data do not indicate a significant difference. The relative radiative ratio $P=5$ should be understood as the ratio between the $\mathrm{CoO}$ (RIXS/Auger) ratio and the Co (FL/ Auger) ratio. Note that if the radiative decay is relatively stronger for $\mathrm{CoO}$ the nonradiative decay should be relatively stronger for Co metal, but now the difference is between $95 \%$ and $99 \%$ of the total decay, which is difficult to determine. A full study of all radiative and nonradiative decay channels for $\mathrm{CoO}$ and $\mathrm{Co}$ metal would be interesting for a fundamental knowledge of the creation and decay of $2 \mathrm{p}$ core holes in oxides and metals, respectively.

For the purposes of the current study, we take the relative radiative ratio $P=5$. The converted $\mathrm{CoO}$ concentrations obtained, by using this number, are shown in the fourth column of Table 1 . These values can be determined analytically from the spectral weight numbers in column 3. Defining the spectral weight as $x$, the atomic ratio $\left(x_{\mathrm{A}}\right)$ can be calculated as $x_{\mathrm{A}}=x /[P$ $\left.+x-\left(P^{*} x\right)\right]$. The sigma values are calculated accordingly. Comparing the relative errors for concentration of the minority phase $\mathrm{CoO}$ from columns 2 and 4, we note that the error from XAS averages to $\sim 5.6 \%$, while in the case of RIXS it averages to $0.8 \%$. Within the present study the RIXS error is affected by the uncertainty in the relative radiative ratio. Once this factor is established, RIXS will become a very accurate technique for the distinction of different phases, especially for the determination of small amounts of oxide phases.

It is evident that fitting results from $2 \mathrm{p} 3 \mathrm{~d}$ RIXS spectra are more precise than those from $2 p$ XAS. In principle, the reported errors reflect primarily the well-resolved characteristic features in RIXS, compared to XAS, as systematic errors get reduced by holistically treating the data. Furthermore, the precision of $\mathrm{CoO}$ phase concentrations by RIXS fitting procedures should also be influenced by the precision of the relative radiative ratio obtained. The obtained value, although approximate in nature, suggests that $2 \mathrm{p} 3 \mathrm{~d}$ RIXS is feasible to probe the minority $\mathrm{CoO}$ phase with higher intrinsic accuracy in RIXS than the metallic phase.

Future studies should determine the generality of the relative radiative ratio or in other words the relative radiative decay rates of different systems. This is in itself an important factor determined by the nonradiative decay and the radiative decay channels. We believe that the general assumption of a fixed lifetime broadening only determined by the core hole is not valid in the case of $2 p$ core holes in $3 \mathrm{~d}$ transition metal systems. The general analysis is complicated by the fact that the radiative decay, and to a lesser extent also the nonradiative decay, is energy dependent.

We note that using RIXS to enhance the accuracy from XAS is a general approach. In fact the case of $\mathrm{CoO}$ and $\mathrm{Co}$ metal is one of the most difficult cases due to the large overlap of both XAS spectra. For example, the distinction between XAS spectra of $\mathrm{Co}^{2+}$ oxides and $\mathrm{Co}^{3+}$ oxides is much larger, allowing a much stronger improvement in the quantitative determination of the amounts of $\mathrm{Co}^{2+}$ and $\mathrm{Co}^{3+}$. In such studies it is important (1) to measure the XAS spectra as accurate as possible and (2) to experimentally determine the relative radiative ratio between $\mathrm{Co}^{2+}$ and $\mathrm{Co}^{3+}$.

\section{CONCLUDING REMARKS}

The fact that $2 \mathrm{p} 3 \mathrm{~d}$ RIXS has (1) a large variation in sensitivities for different phases and (2) can selectively enhance features of a certain phase via resonant excitations creates an advantage over $2 \mathrm{p}$ XAS for studying minority phases. In addition, TEY $2 \mathrm{p}$ XAS is limited by the probing depth, and total fluorescence yield $2 p$ XAS may suffer from distorted spectral shapes ${ }^{29}$ that 
complicate theoretical interpretations. By comparison, $2 \mathrm{p} 3 \mathrm{~d}$ RIXS can detect a bulk phase buried under the surface, and high-resolution spectral features allow more accurate phase determination from theoretical analyses than $2 \mathrm{p}$ XAS. We determined accuracies of, respectively, 5.6\% for XAS and $0.8 \%$ for RIXS. In addition, we have made a first estimate of the relative radiative ratio between $\mathrm{CoO}$ and $\mathrm{Co}$ metal, as being approximately 5 . Further studies need to establish this relative radiative ratio more accurately and generalize its usage to different metal ions. This is the first approach in which multiple chemical phases in an in-situ 2p3d RIXS experiment are effectively decomposed to contributions from individual phases to quantify the corresponding concentrations. By this approach, the minority phase is quantified with much higher precision than that quantified from $2 \mathrm{p}$ XAS results. We note that if the Xray emission resolution in the $2 \mathrm{p} 3 \mathrm{~d}$ RIXS experiment is improved this will further enhance the capabilities of RIXS for chemical selectivity.

\section{ASSOCIATED CONTENT}

\section{S Supporting Information}

The Supporting Information is available free of charge on the ACS Publications website at DOI: 10.1021/acs.jpcc.7b04325.

Synthesis of the CNT-supported Co FT catalyst; XRD of the fresh $\mathrm{Co}_{3} \mathrm{O}_{4} / \mathrm{CNT}$ catalyst; in-situ 2pXAS and 2p3d RIXS measurements; Co $2 \mathrm{p}$ XAS spectra of $\mathrm{CoO}$ and $\mathrm{Co}$ metal mixture with $<10 \% \mathrm{CoO}$ phase concentration; spectral comparison of decompose-fitted CoO RIXS with literature reference $\mathrm{CoO}$ RIXS; decompose-fitted Co metal fluorescence for excitation energies (PDF)

\section{AUTHOR INFORMATION}

\section{Corresponding Authors}

*E-mail: m.u.delgadojaime@uu.nl.

*E-mail: f.m.f.degroot@uu.nl.

ORCID ${ }^{\circ}$

Yi-Tao Cui: 0000-0001-7104-0059

Yoshihisa Harada: 0000-0002-4590-9109

Frank M. F. de Groot: 0000-0002-1340-2186

\section{Author Contributions}

All authors have given approval to the final version of the manuscript.

\section{Notes}

The authors declare no competing financial interest.

\section{ACKNOWLEDGMENTS}

The synchrotron radiation experiments were performed at the HORNET end station of BL07LSU of SPring- 8 with the approval of the Japan Synchrotron Radiation Research Institute (JASRI) (Proposal No. 2013B7458, 2014A7470, 2014A7468). This work was financially supported by the China Scholarship Council and the ERC advanced grant XRAYonACTIVE, number 340279 .

\section{REFERENCES}

(1) Hayakawa, H.; Tanaka, H.; Fujimoto, K. Studies on Precipitated Iron Catalysts for Fischer-Tropsch Synthesis. Appl. Catal., A 2006, $310,24-30$.

(2) Li, S. Z.; Meitzner, G. D.; Iglesia, E. Spectroscopic and Transient Kinetic Studies of Site Requirements in Iron-Catalyzed FischerTropsch Synthesis. J. Phys. Chem. B 2001, 105, 5743-5750.
(3) Takamatsu, D.; Koyama, Y.; Orikasa, Y.; Mori, S.; Nakatsutsumi, T.; Hirano, T.; Tanida, H.; Arai, H.; Uchimoto, Y.; Ogumi, Z. First In Situ Observation of the $\mathrm{LiCoO}_{2}$ Electrode/Electrolyte Interface by Total-Reflection X-ray Absorption Spectroscopy. Angew. Chem., Int. Ed. 2012, 51, 11597-11601.

(4) Bordiga, S.; Groppo, E.; Agostini, G.; van Bokhoven, J. A.; Lamberti, C. Reactivity of Surface Species in Heterogeneous Catalysts Probed by In Situ X-ray Absorption Techniques. Chem. Rev. 2013, 113, 1736-1850.

(5) Groot, F. M. F. Multiplet Effects in X-ray Spectroscopy. Coord. Chem. Rev. 2005, 249, 31-63.

(6) Tuxen, A.; Carenco, S.; Chintapalli, M.; Chuang, C. H.; Escudero, C.; Pach, E.; Jiang, P.; Borondics, F.; Beberwyck, B.; Alivisatos, A. P.; et al. Size-Dependent Dissociation of Carbon Monoxide on Cobalt Nanoparticles. J. Am. Chem. Soc. 2013, 135, 2273-2278.

(7) Morales, F.; de Groot, F. M. F.; Glatzel, P.; Kleimenov, E.; Bluhm, H.; Havecker, M.; Knop-Gericke, A.; Weckhuysen, B. M. In Situ X-ray Absorption of $\mathrm{Co} / \mathrm{Mn} / \mathrm{TiO}_{2}$ Catalysts for Fischer-Tropsch Synthesis. J. Phys. Chem. B 2004, 108, 16201-16207.

(8) Ghiasi, M.; Delgado-Jaime, M. U.; Malekzadeh, A.; Wang, R. P.; Miedema, P. S.; Beye, M.; de Groot, F. M. F. Mn and Co Charge and Spin Evolutions in $\mathrm{LaMn}_{1-\mathrm{x}} \mathrm{Co}_{\mathrm{x}} \mathrm{O}_{3}$ Nanoparticles. J. Phys. Chem. C 2016, 120, 8167-8174.

(9) Lee, H. J.; Kim, G.; Kim, D. H.; Kang, J. S.; Zhang, C. L.; Cheong, S. W.; Shim, J. H.; Lee, S.; Lee, H.; Kim, J. Y.; et al. Valence States and Occupation Sites in $(\mathrm{Fe}, \mathrm{Mn})_{3} \mathrm{O}_{4}$ Spinel Oxides Investigated by Soft $\mathrm{X}$-ray Absorption Spectroscopy and Magnetic Circular Dichroism. J. Phys.: Condens. Matter 2008, 20, 295203.

(10) van Schooneveld, M. M.; Suljoti, E.; Campos-Cuerva, C.; Gosselink, R. W.; van der Eerden, A. M. J.; Schlappa, J.; Zhou, K. J. J.; Monney, C.; Schmitt, T.; de Groott, F. M. F. Transition-Metal Nanoparticle Oxidation in a Chemically Nonhomogenous Environment Revealed by 2p3d Resonant X-ray Emission. J. Phys. Chem. Lett. 2013, 4, 1161-1166.

(11) Eschemann, T. O.; Lamme, W. S.; Manchester, R. L.; Parmentier, T. E.; Cognigni, A.; Ronning, M.; de Jong, K. P. Effect of Support Surface Treatment on the Synthesis, Structure, and Performance of Co/CNT Fischer-Tropsch Catalysts. J. Catal. 2015, $328,130-138$.

(12) Cats, K. H.; Gonzalez-Jimenez, I. D.; Liu, Y. J.; Nelson, J.; van Campen, D.; Meirer, F.; van der Eerden, A. M. J.; de Groot, F. M. F.; Andrews, J. C.; Weckhuysen, B. M. X-ray Nanoscopy of Cobalt Fischer-Tropsch Catalysts at Work. Chem. Commun. 2013, 49, 46224624.

(13) Tsakoumis, N. E.; Dehghan, R.; Johnsen, R. E.; Voronov, A.; van Beek, W.; Walmsley, J. C.; Borg, O.; Rytter, E.; Chen, D.; Ronning, M.; et al. Combined In Situ XAS-XRPD-Raman Study of Fischer-Tropsch Synthesis Over a Carbon Supported Co Catalyst. Catal. Today 2013, 205, 86-93.

(14) Chen, W.; Pan, X. L.; Willinger, M. G.; Su, D. S.; Bao, X. H. Facile Autoreduction of Iron Oxide/Carbon Nanotube Encapsulates. J. Am. Chem. Soc. 2006, 128, 3136-3137.

(15) Xiong, H. F.; Moyo, M.; Rayner, M. K.; Jewell, L. L.; Billing, D. G.; Coville, N. J. Autoreduction and Catalytic Performance of a Cobalt Fischer-Tropsch Synthesis Catalyst Supported on Nitrogen-Doped Carbon Spheres. ChemCatChem 2010, 2, 514-518.

(16) Xiong, H. F.; Motchelaho, M. A. M.; Moyo, M.; Jewell, L. L.; Coville, N. J. Correlating the Preparation and Performance of Cobalt Catalysts Supported on Carbon Nanotubes and Carbon Spheres in the Fischer-Tropsch Synthesis. J. Catal. 2011, 278, 26-40.

(17) Harada, Y.; Kobayashi, M.; Niwa, H.; Senba, Y.; Ohashi, H.; Tokushima, T.; Horikawa, Y.; Shin, S.; Oshima, M. Ultrahigh Resolution Soft X-ray Emission Spectrometer at BL07LSU in SPring-8. Rev. Sci. Instrum. 2012, 83, 013116.

(18) van Schooneveld, M. M.; Kurian, R.; Juhin, A.; Zhou, K. J.; Schlappa, J.; Strocov, V. N.; Schmitt, T.; de Groot, F. M. F. Electronic Structure of $\mathrm{CoO}$ Nanocrystals and a Single Crystal Probed by Resonant X-ray Emission Spectroscopy. J. Phys. Chem. C 2012, 116, $15218-15230$. 
(19) Chen, C. T.; Idzerda, Y. U.; Lin, H. J.; Smith, N. V.; Meigs, G.; Chaban, E.; Ho, G. H.; Pellegrin, E.; Sette, F. Experimental Confirmation of the X-Ray Magnetic Circular Dichroism Sum Rules for Iron and Cobalt. Phys. Rev. Lett. 1995, 75, 152-155.

(20) Kotani, A.; Shin, S. Resonant Inelastic X-ray Scattering Spectra for Electrons in Solids. Rev. Mod. Phys. 2001, 73, 203-246.

(21) Ament, L. J. P.; van Veenendaal, M.; Devereaux, T. P.; Hill, J. P.; van den Brink, J. Resonant Inelastic X-ray Scattering Studies of Elementary Excitations. Rev. Mod. Phys. 2011, 83, 705-767.

(22) Magnuson, M.; Butorin, S. M.; Guo, J. H.; Nordgren, J. Electronic Structure Investigation of $\mathrm{CoO}$ by Means of Soft X-ray Scattering. Phys. Rev. B: Condens. Matter Mater. Phys. 2002, 65, 205106.

(23) van Schooneveld, M. M.; Juhin, A.; Campos-Cuerva, C.; Schmitt, T.; de Groot, F. M. F. Origin of Low Energy d-d Excitations Observed on Wet Chemically Prepared Cobalt Bearing Nanoparticles by 2 p3d Resonant X-ray Emission Spectroscopy. J. Phys. Chem. C 2013, 117, 14398-14407.

(24) Seah, M. P.; Dench, W. A. Quantitative Electron Spectroscopy of Surfaces: A Standard Data Base for Electron Inelastic Mean Free Paths in Solids. Surf. Interface Anal. 1979, 1, 2-11.

(25) Henke, B. L.; Gullikson, E. M.; Davis, J. C. X-Ray Interactions: Photoabsorption, Scattering, Transmission, and Reflection at $\mathrm{E}=50-$ 30,000 eV, Z = 1-92. At. Data Nucl. Data Tables 1993, 54, 181-342. (26) Delgado-Jaime, M. U.; Kennepohl, P. Development and Exploration of a New Methodology for the Fitting and Analysis of XAS Data. J. Synchrotron Radiat. 2010, 17, 119-128.

(27) Delgado-Jaime, M. U.; Mewis, C. P.; Kennepohl, P. Blueprint XAS: A Matlab-based Toolbox for the Fitting and Analysis of XAS Spectra. J. Synchrotron Radiat. 2010, 17, 132-137.

(28) de Groot, F. M. F.; Pizzini, S.; Fontaine, A.; Hamalainen, K.; Kao, C. C.; Hastings, J. B. Local-spin-selective X-ray Absorption and X-ray Magnetic Circular Dichroism of MnP. Phys. Rev. B: Condens. Matter Mater. Phys. 1995, 51, 1045-1052.

(29) de Groot, F. M. F.; Arrio, M. A.; Sainctavit, P.; Cartier, C.; Chen, C. T. Fluorescence Yield Detection: Why It Does Not Measure the X-ray Absorption Cross Section. Solid State Commun. 1994, 92, 991-995. 